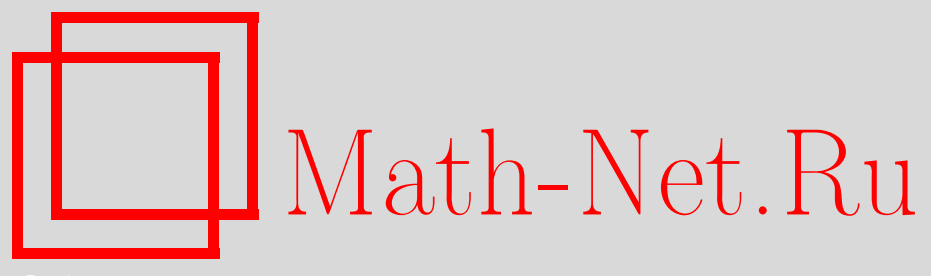

Н. Н. Пустовойтов, О приближении периодических функций из классов $H_{q}^{\Omega}$ линейными методами, Матем. сб., 2012, том 203, номер 1, 91-114

DOI: https://doi.org/10.4213/sm7694

Использование Общероссийского математического портала Math-Net.Ru подразумевает, что вы прочитали и согласны с пользовательским соглашением http://www.mathnet.ru/rus/agreement

Параметры загрузки:

IP: 34.239 .49 .27

26 апреля 2023 г., 18:06:16

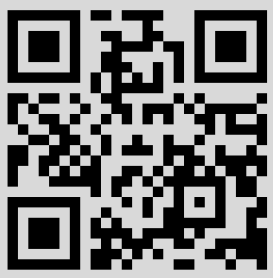




\section{Н. Н. Пустовойтов}

\section{О приближении периодических функций из классов $H_{q}^{\Omega}$ линейными методами}

В работе показано, что если приближения функций из классов $H_{\infty}^{\Omega}$ (из классов $H_{1}^{\Omega}$ ) в норме $L_{\infty}$ (в норме $H_{1}$ ) линейными операторами имеют порядок наилучших приближений, то нормы этих операторов не ограничены в совокупности. Также установлены неравенства Бернштейна и Джексона-Никольского для тригонометрических полиномов со спектром из множеств $Q(N)$ (из множеств $\Gamma(N, \Omega)$ ).

Библиография: 15 названий.

Ключевые слова: модуль непрерывности, линейные приближения, неравенства Бернштейна, неравенства Никольского, функции многих переменных.

\section{Введение}

В работе изучается возможность приближения периодических функций многих переменных из классов $H_{q}^{\Omega}$ линейными методами, дающими наилучшие по порядку приближения. А именно выясняется, существуют ли линейные ограниченные операторы, дающие приближения порядка наилучших и такие, что их нормы ограничены в совокупности. Для классов Никольского $H_{q}^{r}$ и Соболева $W_{q, \alpha}^{r}$ эти вопросы изучались В.Н. Темляковым в [1].

Для классов Бесова $B_{p, \theta}^{r}$ указанные вопросы изучались А.С. Романюком в [2].

\section{Дадим определения.}

Для $t=\left(t_{1}, \ldots, t_{d}\right), t_{j} \geqslant 0, j=1, \ldots, d$, определим функцию $\Omega(t)$ следующим образом: если $t_{j}>0, j=1, \ldots, d$, то

$$
\Omega(t)=\prod_{j=1}^{d} t_{j}^{r}\left(\log \frac{1}{t_{j}}\right)_{+}^{-b_{j}},
$$

если $\prod_{j=1}^{d} t_{j}=0$, то $\Omega(t)=0 ;$ здесь

$$
\left(\log \frac{1}{t_{j}}\right)_{+}=\max \left\{\log _{2} \frac{1}{t_{j}} ; 1\right\}, \quad 0<r<l,
$$

где $l$ - порядок разностей (см. определение $\left.\Delta_{t}^{l} f(x)\right)$.

Полагаем, что $b_{1} \leqslant \cdots \leqslant b_{d}$. 
Легко видеть, что при $0 \leqslant t_{j} \leqslant 2^{\frac{b_{j}}{r}}$ функция $\Omega(t)$ возрастает по каждой переменной. Нас интересует поведение функции $\Omega(t)$ вблизи нуля, а при $t_{j}>2^{\frac{b_{j}}{r}}$ эту функцию можно доопределить, положив $\Omega(t)=\Omega\left(2^{\frac{b_{1}}{r}}, \ldots, 2^{\frac{b_{d}}{r}}\right)$.

Рассмотрим множества

$$
\begin{gathered}
\varkappa(N)=\left\{s=\left(s_{1}, \ldots, s_{d}\right): s_{j} \in \mathbb{N}, j=1, \ldots, d, \prod_{j=1}^{d} 2^{r s_{j}} s_{j}^{b_{j}} \leqslant N\right\}, \\
Q(N)=\bigcup_{s \in \varkappa(N)} \rho(s),
\end{gathered}
$$

где

$$
\rho(s)=\left\{k=\left(k_{1}, \ldots, k_{d}\right): k_{j} \in \mathbb{Z}, 2^{s_{j}-1} \leqslant\left|k_{j}\right|<2^{s_{j}}, j=1, \ldots, d\right\} .
$$

Также введем множества

$$
\Gamma(N, \Omega)=\left\{k=\left(k_{1}, \ldots, k_{d}\right):\left|k_{j}\right| \in \mathbb{N}, j=1, \ldots, d, \Omega\left(\frac{1}{\left|k_{1}\right|}, \ldots, \frac{1}{\left|k_{d}\right|}\right) \geqslant \frac{1}{N}\right\},
$$

т.e.

$$
\Gamma(N, \Omega)=\left\{k=\left(k_{1}, \ldots, k_{d}\right):\left|k_{j}\right| \in \mathbb{N}, j=1, \ldots, d, \prod_{j=1}^{d}\left|k_{j}\right|^{r}\left(\log \left|k_{j}\right|\right)_{+}^{b_{j}} \leqslant N\right\} .
$$

Легко видеть, что $Q(N) \subset \Gamma(N, \Omega) \subset Q\left(2^{r d+\sum_{j=1}^{d}\left|b_{j}\right|}\right)$.

Положим

$$
\theta(N)=\left\{s=\left(s_{1}, \ldots, s_{d}\right): s_{j} \in \mathbb{N}, j=1, \ldots, d, N<\prod_{j=1}^{d} 2^{r s_{j}} s_{j}^{b_{j}} \leqslant 2^{l} N\right\} .
$$

Как легко видеть, $\theta(N)=\varkappa\left(2^{l} N\right) \backslash \varkappa(N)$.

Мы здесь изучаем порядковые равенства и неравенства.

Запись $A(N) \ll B(N)$ означает, что существует $C>0$, не зависящее от $N$, такое, что $A(N) \leqslant C \cdot B(N)$. Запись $A(N) \asymp B(N)$ означает, что $A(N) \ll$ $B(N) \ll A(N)$.

Автором в [3] доказано, что количество элементов множества $\theta(N)$ по порядку равно $|\theta(N)| \asymp(\log N)^{d-1}$.

Также автором доказана следующая теорема, формулировка которой приведена в [4].

Теорема 0.1. Количество элементов множеств $Q(N)$ и $\Gamma(N, \Omega)$ по порядку равно:

1) $N^{\frac{1}{r}}(\log N)^{d-1-\frac{1}{r}\left(b_{1}+\cdots+b_{d}\right)}$, если $b_{d}<r$;

2) $N^{\frac{1}{r}}(\log N)^{\nu-1-\frac{1}{r}\left(b_{1}+\cdots+b_{\nu}\right)}(\log \log N)^{\mu}$, если $b_{\nu}<r=b_{\nu+1}=\cdots=b_{\nu+\mu}<$ $b_{\nu+\mu+1}$

3) $N^{\frac{1}{r}}(\log N)^{-1}(\log \log N)^{\nu-1}$, если $r=b_{1}=\cdots=b_{\nu}<b_{\nu+1}$;

4) $N^{\frac{1}{r}}(\log N)^{-\frac{b_{1}}{r}}$, если $b_{2}>r$. 
Мы будем рассматривать классы $H_{q}^{\Omega}$ функций $d$ вещественных переменных, имеющих период $2 \pi$ по каждой переменной и удовлетворяющих условиям:

1) $f(x)=f\left(x_{1}, \ldots, x_{d}\right) \in L_{q}^{0}$, т.е. норма

$$
\begin{gathered}
\|f(x)\|_{q}=\left((2 \pi)^{-d} \int_{\pi_{d}}|f(x)|^{q} d x\right)^{\frac{1}{q}} \quad \text { при } 1 \leqslant q<\infty, \\
\|f(x)\|_{\infty}=\text { sup vrai }|f(x)| \quad \text { при } q=\infty
\end{gathered}
$$

конечна и, кроме того,

$$
\int_{-\pi}^{\pi} f(x) d x_{j}=0, \quad j=1, \ldots, d, \quad \pi_{d}=[-\pi ; \pi]^{d} ;
$$

2) $\left\|\Delta_{t}^{l} f(x)\right\|_{q} \leqslant \Omega(t)$, где $\Omega(t)$ определено в (1), а разность

$$
\Delta_{t}^{l} f(x)=\Delta_{t_{1}}^{l} \cdots \Delta_{t_{d}}^{l} f(x)
$$

- это смешанная разность порядка $l$, т.е. берется разность порядка $l$ по переменной $x_{j}$ с шагом $t_{j}$.

Для классов $H_{q}^{\Omega}$ в работе [5] доказана теорема о представлении, которая в случае $\Omega(t)$ из (1) верна и формулируется следующим образом.

ТЕОРема 0.2. Функиия $f(x)$ из $L_{q}^{0}$ принадлежит классу $H_{q}^{\Omega}$ c $\Omega(t)$ из (1) тогда и только тогда, когда для всех векторов $s=\left(s_{1}, \ldots, s_{d}\right)$ с натуральньми компонентами верны неравенства

$$
\begin{aligned}
\left\|\delta_{s}(f, x)\right\|_{q} \ll \Omega\left(2^{-s}\right), & & 1<q<\infty, \\
\left\|A_{s}(f, x)\right\|_{q} \ll \Omega\left(2^{-s}\right), & & 1 \leqslant q \leqslant \infty .
\end{aligned}
$$

Здесь

$$
\begin{gathered}
\delta_{s}(f, x)=\sum_{k \in \rho(s)} \widehat{f}(k) e^{i(k, x)}, \quad(k, x)=k_{1} x_{1}+\cdots+k_{d} x_{d}, \\
A_{s}(f, x)=f(x) * A_{s}(x),
\end{gathered}
$$

где

$$
\begin{gathered}
A_{s}(x)=2^{d} \prod_{j=1}^{d} A_{s_{j}}\left(x_{j}\right), \quad A_{0}\left(x_{j}\right) \equiv 1, \quad A_{1}\left(x_{j}\right)=V_{1}\left(x_{j}\right)-1, \\
A_{s_{j}}\left(x_{j}\right)=V_{2^{s_{j}-1}}\left(x_{j}\right)-V_{2^{s_{j}-2}}\left(x_{j}\right), \quad s_{j} \geqslant 2 ;
\end{gathered}
$$

$V_{m}(\tau)$ - одномерное ядро Валле Пуссена порядка $2 m-1$.

Автором доказана следующая лемма, формулировка которой приведена в [4]. Лемма 0.1. Сумма

$$
\sum_{s \in \theta(N)} \prod_{j=1}^{d} s_{j}^{-\gamma_{j}}, \quad \gamma_{1} \leqslant \cdots \leqslant \gamma_{d}
$$

по порядку равна: 
1) $(\log N)^{d-1-\gamma_{1}-\cdots-\gamma_{d}}$, ecлu $\gamma_{d}<1$;

2) $(\log N)^{\nu-1-\gamma_{1}-\cdots-\gamma_{\nu}}(\log \log N)^{\mu}$, если $\gamma_{\nu}<1=\gamma_{\nu+1}=\cdots=\gamma_{\nu+\mu}<$ $\gamma_{\nu+\mu+1}$

3) $(\log N)^{-1}(\log \log N)^{\nu-1}$, если $\gamma_{1}=\cdots=\gamma_{\nu}=1<\gamma_{\nu+1}$;

4) $(\log N)^{-\gamma_{1}}$, если $1<\gamma_{1}$.

Сформулируем две леммы, которые будут нужны нам в дальнейшем (см. [1; леммы 1.3.1, 1.3.1']).

Лемма 0.2. Пусть $1 \leqslant q<p<\infty, f \in L_{q}^{0}\left(\pi_{d}\right)$. Тогда

$$
\|f\|_{p}^{p} \ll \sum_{s}\left(\left\|\delta_{s}(f)\right\|_{q} 2^{\|s\|_{1}\left(\frac{1}{q}-\frac{1}{p}\right)}\right)^{p} .
$$

Лемма 0.3. Пусть $1<p<q \leqslant \infty, f \in L_{p}^{0}\left(\pi_{d}\right)$. Тогда

$$
\|f\|_{p}^{p} \gg \sum_{s}\left\|\delta_{s}(f)\right\|_{q}^{p} 2^{\|s\|_{1}\left(\frac{1}{q}-\frac{1}{p}\right) p}
$$

В $\S 1$ доказываются неравенства Бернштейна, а в 2 доказываются неравенства Джексона-Никольского для тригонометрических полиномов со спектром из $\Gamma(N, \Omega)$. Эти результаты носят вспомогательный характер, хотя могут иметь и самостоятельный интерес.

В $\S 3$ доказывается, что при некоторых условиях на $b_{j}$ нельзя построить последовательность линейных ограниченных операторов, дающих приближения порядка наилучших функций из классов $H_{\infty}^{\Omega}$ в норме $L_{\infty}$ и классов $H_{1}^{\Omega}$ в норме $L_{1}$ таких, что нормы операторов ограничены в совокупности.

\section{$\S 1$. Неравенства Бернштейна}

Через $T(\mathrm{~A})$ будем обозначать множество тригонометрических полиномов со спектром из множества А.

Для $t(x) \in T(\Gamma(N, \Omega))$ определим производную следующим образом:

$$
t^{(\rho)}(x, \alpha)=t * U_{N}^{\rho},
$$

где

$$
\begin{gathered}
U_{N}^{\rho}=\sum_{k \in \Gamma(N, \Omega)} \prod_{j=1}^{d} k_{j}^{\rho} \cos \left(k_{j} x_{j}+\frac{\alpha_{j} \pi}{2}\right), \\
\rho \geqslant 0, \quad \alpha=\left(\alpha_{1}, \ldots, \alpha_{d}\right), \quad \alpha_{j} \in \mathbb{R}, \quad j=1, \ldots, d .
\end{gathered}
$$

Теорема 1.1. 1) При $1<p<\infty$ верно порядковое равенство

$$
\sup _{t \in T(\Gamma(N, \Omega))} \frac{\left\|t^{(\rho)}(x, \alpha)\right\|_{p}}{\|t\|_{p}} \asymp N^{\frac{\rho}{r}}(\log N)^{-\frac{b_{1} \rho}{r}} .
$$


2) При $p=\infty$ величина

$$
\sup _{t \in T(\Gamma(N, \Omega))} \frac{\left\|t^{(\rho)}(x, \alpha)\right\|_{\infty}}{\|t\|_{\infty}}
$$

по порядку равна

$$
\begin{aligned}
& N^{\frac{\rho}{r}}(\log N)^{d-1-\frac{\rho}{r}\left(b_{1}+\cdots+b_{d}\right)}, \quad \text { ecлu } \quad b_{d}<\frac{r}{\rho}, \\
& N^{\frac{\rho}{r}}(\log N)^{\nu-1-\frac{\rho}{r}\left(b_{1}+\cdots+b_{\nu}\right)}(\log \log N)^{\mu}, \quad \text { ecлu } b_{\nu}<\frac{r}{\rho}=b_{\nu+1}=\cdots=b_{\nu+\mu} \\
& <b_{\nu+\mu+1} \\
& N^{\frac{\rho}{r}} \frac{(\log \log N)^{\nu-1}}{\log N}, \quad \text { если } b_{1}=\cdots=b_{\nu}=\frac{r}{\rho}<b_{\nu+1}, \\
& N^{\frac{\rho}{r}}(\log N)^{-\frac{b_{1} \rho}{r}}, \quad \text { ecлu } b_{2}>\frac{r}{\rho} .
\end{aligned}
$$

Неравенства Бернштейна для тригонометрических полиномов со спектром из гиперболических крестов изучались в [1], [6]-[10]. К этому кругу вопросов примыкает работа [11]. В [12] М. Б. Сихов при $1<p<\infty$ получил неравенства Бернштейна для $t \in T(\Gamma(N, \Omega))$ в случае двух переменных при $b_{1} \leqslant 0, b_{2}=0$, а также при $b_{1} \leqslant 0, b_{2}(p-1)>r>0$.

ДокаЗАТЕЛЬСтво теоремы 1.1. Сначала рассмотрим случай $1<p<\infty$.

Как и при доказательстве теоремы 1.1.1 в [1], используем множители Марцинкевича $\lambda_{\nu}^{\rho}=|\nu|^{\rho} 2^{-\rho s}, 2^{s-1} \leqslant|\nu|<2^{s}$. Как показано в [1], для мультипликаторного оператора $\Lambda_{\rho}$, задаваемого множителями $\lambda_{\nu}^{\rho}$, будет $\left\|\Lambda_{\rho} f\right\|_{p} \asymp|f|_{p}$. Далее, поскольку оператор тригонометрического сопряжения ограничен в $L_{p}$, $1<p<\infty$, то без ограничения общности можно считать $\alpha_{j}=0, j=1, \ldots, d$.

Имеем

$$
\left\|t^{(\rho)}(x, 0)\right\|_{p} \asymp\left\|\Lambda_{p}^{-1} t^{(\rho)}\right\|_{p} \asymp\left\|\left(\sum_{s \in \varkappa\left(N_{1}\right)} 2^{2 \rho\|s\|_{1}}\left|\delta_{s}(t, x)\right|^{2}\right)^{\frac{1}{2}}\right\|_{p} .
$$

Здесь

$$
N_{1}=2^{r d+\sum_{j=1}^{d}\left|b_{j}\right|} N, \quad\|s\|_{1}=s_{1}+\cdots+s_{d}
$$

и, кроме того,

$$
\delta_{s}(t, x)=\sum_{k \in \rho(s)} \widehat{t}(k) e^{i(k, x)},
$$

где $(k, x)=k_{1} x_{1}+\cdots+k_{d} x_{d} ; \widehat{t}(k)-$ коэффициенты Фурье. Кроме того, здесь применена многомерная теорема Литтлвуда-Пэли для функций многих переменных (см. [1; теорема А]). Далее,

$$
\left\|t^{(\rho)}(x, 0)\right\|_{p} \ll \max _{s \in \varkappa\left(N_{1}\right)} 2^{\rho\|s\|_{1}}\|t\|_{p} \ll N^{\frac{\rho}{r}}(\log N)^{-\frac{b_{1} \rho}{r}}\|t\|_{p} .
$$

Оценка сверху доказана. 
Для доказательства оценки снизу при $1<p<\infty$ возьмем тригонометрический полином $t(x)=e^{i\left(2^{\widetilde{s}}, x\right)}$, где

$$
\begin{gathered}
\widetilde{s}=\left(\widetilde{s}_{1}, \ldots, \widetilde{s}_{d}\right), \\
\widetilde{s}_{j}= \begin{cases}\max _{s \in \varkappa\left(N_{1}\right)} s_{1}, & j=1, \\
1, & j=2,3, \ldots, d .\end{cases}
\end{gathered}
$$

Тогда

$$
\left\|t^{(\rho)}(x, 0)\right\|_{p} \asymp 2^{\rho \widetilde{s}_{1}}\|t\|_{p} \asymp N^{\frac{\rho}{r}}(\log N)^{-\frac{b_{1} \rho}{r}}\|t\|_{p} .
$$

Далее рассмотрим случай $p=\infty$.

По аналогии с доказательством теоремы 1.1 .1 в [1] рассмотрим функцию

$$
K_{N}^{\rho}(x, \alpha)=\sum_{s \in \varkappa\left(2^{k l} N\right)} A_{s}^{\rho}(x, \alpha)
$$

где $k$ выбрано так, что $2^{k l} \geqslant 2^{r d} 3^{\sum_{j=1}^{d}\left|b_{j}\right|}(l-$ порядок разности). Кроме того,

$$
\begin{gathered}
A_{s}^{\rho}(x, \alpha)=\prod_{j=1}^{d} A_{s_{j}}^{\rho}\left(x_{j}, \alpha_{j}\right), \quad s=\left(s_{1}, \ldots, s_{d}\right), \quad s_{j} \in \mathbb{N}, \quad s_{j} \geqslant 2, \quad j=1, \ldots, d, \\
A_{s_{j}}^{\rho}\left(x_{j}, \alpha_{j}\right)=V_{2^{s_{j}-1}}^{\rho}\left(x_{j}, \alpha_{j}\right)-V_{2^{s_{j}-2}}^{\rho}\left(x_{j}, \alpha_{j}\right), \\
V_{m}^{\rho}\left(x_{j}, \alpha_{j}\right)=\sum_{k=1}^{m} k^{\rho} \cos \left(k x_{j}+\frac{\alpha_{j} \pi}{2}\right)+\sum_{k=m+1}^{2 m-1} k^{\rho}\left(1-\frac{k-m}{m}\right) \cos \left(k x_{j}+\frac{\alpha_{j} \pi}{2}\right) .
\end{gathered}
$$

Тогда для $t(x)$ из $T(\Gamma(N, \Omega))$ будет $t^{(\rho)}(x, \alpha)=t * K_{N}^{\rho}(x, \alpha)$. Следовательно,

$$
\left\|t^{(\rho)}(x, \alpha)\right\|_{\infty} \leqslant\|t\|_{\infty} \cdot\left\|K_{N}^{\rho}\right\|_{1} .
$$

Получаем

$$
\left\|K_{N}^{\rho}\right\|_{1} \ll \sum_{\nu=1}^{\left[\frac{1}{l} \log N\right]} \sum_{s \in \theta\left(\frac{N}{2^{k \nu}}\right)}\left\|A_{s}^{\rho}(x, \alpha)\right\|_{1} \ll \sum_{s \in \theta(N)} 2^{\rho\|s\|_{1}} \asymp N^{\frac{\rho}{r}} \sum_{s \in \theta(N)} \prod_{j=1}^{d} s_{j}^{-\frac{b_{j} \rho}{r}} .
$$

Применяя лемму 0.1 с $\gamma_{j}=\frac{b_{j} \rho}{r}$, получаем оценку сверху.

Для получения оценки снизу используем функцию

$$
\bar{U}_{N}^{\beta}(x, \alpha)=\sum_{k \in \Gamma(N, \Omega)} \prod_{j=1}^{d} a\left(k_{j}, x_{j}, \alpha_{j}\right) k_{j}^{\beta},
$$

где

$$
\begin{aligned}
a\left(k_{j}, x_{j}, \alpha_{j}\right)=\cos & \left(k_{j} x_{j}+\frac{\alpha_{j} \pi}{2}\right)+\cos \left(\left(k_{j}+1\right) x_{j}+\frac{\alpha_{j} \pi}{2}\right) \\
& \quad-\cos \left(2 k_{j} x_{j}+\frac{\alpha_{j} \pi}{2}\right)-\cos \left(\left(2 k_{j}+1\right) x_{j}+\frac{\alpha_{j} \pi}{2}\right) .
\end{aligned}
$$


При $\beta=-1$ для гиперболических крестов эти функции построил С. А. Теляковский (см. [7]). При произвольных $\beta \geqslant-1$ эти функции рассмотрел В. Н. Темляков (см. [1; лемма 1.2.1]).

По лемме 1.1.1 из [1]

$$
\left\|\bar{U}_{N}^{-1}(x, \alpha)\right\|_{\infty} \ll 1
$$

Тогда

$$
\left|\left(\bar{U}_{N}^{-1}\right)^{\rho}(0, \alpha)\right| \asymp \sum_{k \in \Gamma(N, \Omega)} \prod_{j=1}^{d} k_{j}^{\rho-1} \gg \sum_{s \in \theta(N)} 2^{\|s\|_{1}(\rho-1+1)}
$$

и мы получаем оценку снизу. Теорема 1.1 доказана.

\section{§ 2. Неравенства Джексона-Никольского}

Рассмотрим неравенства, связывающие различные $L_{p}$-нормы тригонометрических полиномов из $T(\Gamma(N, \Omega))$. Для тригонометрических полиномов со спектром из параллелепипедов, т.е. имеющих степень $n_{j}$ по переменной $x_{j}$, в [13] С. М. Никольский доказал, что

$$
\|t\|_{p} \leqslant 2^{d} \prod_{j=1}^{d} n_{j}^{\frac{1}{q}-\frac{1}{p}} \cdot\|t\|_{q}, \quad 1 \leqslant q<p \leqslant \infty .
$$

Неравенства Джексона-Никольского для случая гиперболических крестов рассмотрены в [1] и [14]. В [15] рассмотрены неравенства Джексона-Никольского для тригонометрических полиномов с заданным числом гармоник. Для тригонометрических полиномов из $T(\Gamma(N, \Omega))$ в случае двух переменных при $1<p<q<\infty, b_{1} \leqslant 0, b_{2}(p-1)>r>0$ неравенства Джексона-Никольского получены в [12].

Докажем леммы, аналогичные соответствующим леммам из [1; гл. 1, п. 2].

Лемма 2.1. Верно порядковое неравенство

$$
\left\|\bar{U}_{N}^{\rho}(x, \alpha)\right\|_{\infty} \ll N^{\frac{\rho+1}{r}}(\log N)^{\frac{-b_{1}(\rho+1)}{r}} \equiv E(N, \infty),
$$

где $\bar{U}_{N}^{\rho}(x, \alpha)-$ функиия из $(3), \rho \geqslant-1$.

ДокАЗАТЕЛЬСтво. По теореме двойственности имеем

$$
\begin{aligned}
\left\|\bar{U}_{N}^{\rho}(x, \alpha)\right\|_{\infty} & =\sup _{\|g\|_{1} \leqslant 1}\left(\bar{U}_{N}^{\rho}, g\right) \asymp \sup _{\|g\|_{1} \leqslant 1} \sum_{\nu=1}^{\left[\frac{1}{l} \log N\right]} \sum_{s \in \theta\left(\frac{N}{2^{l \nu}}\right)}\left(\delta_{s}\left(\bar{U}_{N}^{\rho}\right), g\right) \\
& \ll \sup _{\|g\|_{1} \leqslant 1} \sum_{\nu=1}^{\left[\frac{1}{l} \log N\right]} \sum_{s \in \theta\left(\frac{N}{2^{l \nu}}\right)} 2^{\|s\|_{1}(\rho+1)}\left(\delta_{s}\left(\bar{U}_{N}^{-1}\right), g\right) \\
& \ll \sum_{s \in \theta\left(N_{1}\right)} 2^{\|s\|_{1}(\rho+1)} \cdot\left\|\bar{U}_{N}^{-1}\right\|_{\infty} \asymp N^{\frac{\rho+1}{r}}(\log N)^{\frac{-b_{1}(\rho+1)}{r}} .
\end{aligned}
$$

Здесь $\delta_{s}(f)$ - функция из $(2), N_{1}=2^{r d+\sum_{j=1}^{d}\left|b_{j}\right|} \cdot N$ и использовано неравенство (4). Лемма доказана. 
Лемма 2.2. Пусть $2 \leqslant p<\infty, \rho>\frac{1}{p}-1$. Тогда

$$
\left\|\bar{U}_{N}^{\rho}(x, \alpha)\right\|_{p} \ll E(N, p),
$$

где величина $E(N, p)$ по порядку равна:

1) $N^{\frac{1}{r}\left(\rho+1-\frac{1}{p}\right)}(\log N)^{\frac{d-1}{p}-\frac{1}{r}\left(\rho+1-\frac{1}{p}\right)\left(b_{1}+\cdots+b_{d}\right)}$, если $b_{d}<\frac{r}{p \rho+p-1}$;

2) $N^{\frac{1}{r}\left(\rho+1-\frac{1}{p}\right)}(\log N)^{\frac{\nu-1}{p}-\frac{1}{r}\left(\rho+1-\frac{1}{p}\right)\left(b_{1}+\cdots+b_{\nu}\right)}(\log \log N)^{\frac{\mu}{p}}$, если $b_{\nu}<\frac{r}{p \rho+p-1}=$ $b_{\nu+1}=\cdots=b_{\nu+\mu}<b_{\nu+\mu+1}$;

3) $N^{\frac{1}{r}\left(\rho+1-\frac{1}{p}\right)}(\log N)^{-\frac{1}{p}}(\log \log N)^{\frac{\nu-1}{p}}$, ecлu $b_{1}=\cdots=b_{\nu}=\frac{r}{p \rho+p-1}<b_{\nu+1}$;

4) $N^{\frac{1}{r}\left(\rho+1-\frac{1}{p}\right)}(\log N)^{-\frac{b_{1}}{r}\left(\rho+1-\frac{1}{p}\right)}$, ecлu $b_{2}>\frac{r}{p \rho+p-1}$.

ДокАзАтЕЛЬСтво. Применим лемму 0.2 , считая $q=2, p \geqslant 2$. Получаем

$$
\begin{aligned}
\left\|\bar{U}_{N}^{\rho}\right\|_{p}^{p} & \ll \sum_{\nu=1}^{\left[\frac{1}{l} \log N\right]} \sum_{s \in \theta\left(\frac{N}{2^{l \nu}}\right)}\left\|\delta_{s}\left(\bar{U}_{N}^{\rho}\right)\right\|_{2}^{p} \cdot 2^{\left\|s_{1}\right\|\left(\frac{1}{2}-\frac{1}{p}\right) p} \\
& \asymp \sum_{\nu=1}^{\left[\frac{1}{l} \log N\right]} \sum_{s \in \theta\left(\frac{N}{2^{l \nu}}\right)} 2^{\left\|s_{1}\right\|\left(\left(\rho+\frac{1}{2}\right) p+\left(\frac{1}{2}-\frac{1}{p}\right) p\right)} \\
& \asymp\left|\rho+1-\frac{1}{p}>0\right| \asymp N^{\frac{p}{r}\left(\rho+1-\frac{1}{p}\right)} \sum_{s \in \theta(N)} \prod_{j=1}^{d} s_{j}^{-\frac{b_{j}}{r} p\left(\rho+1-\frac{1}{p}\right)} .
\end{aligned}
$$

Далее, применяя лемму 0.1 с $\gamma_{j}=\frac{b_{j} p}{r}\left(\rho+1-\frac{1}{p}\right)$, получаем лемму 2.2 .

Обозначим

$$
U_{N}^{\rho}(x, \alpha)=\sum_{k \in \Gamma(N, \Omega)} \prod_{j=1}^{d} k_{j}^{\rho} \cos \left(k_{j} x_{j}+\frac{\alpha_{j} \pi}{2}\right) .
$$

Через $E_{\Gamma(N, \Omega)}^{\perp}\left(U_{N}^{\rho}\right)_{p}$ обозначим наилучшее (в норме $\left.L_{p}\right)$ приближение функции $U_{N}^{\rho}(x, \alpha)$ функциями вида

$$
\sum_{k \notin \Gamma(N, \Omega)} c_{k} e^{i(k, x)} .
$$

Лемма 2.3. Пусть $2 \leqslant p \leqslant \infty$. Верно следующее неравенство:

$$
E_{\Gamma(N, \Omega)}^{\perp}\left(U_{N}^{\rho}\right)_{p} \ll E(N, p),
$$

где $\rho>-\frac{1}{2}$ при $2 \leqslant p<\infty u \rho>-1$ при $p=\infty$, a $E(N, p)$ определено в лем$\max 2.1$ u 2.2 .

ДокАЗАТЕЛЬство. Проведем его по индукции по размерности пространства аналогично доказательству лемм 1.2 .3 и $1.2 .3^{\prime}$ из [1]. 
Введем по аналогии с [1] функцию

$$
U(G, d, x)=\sum_{k \in \Gamma(N, \Omega)}\left(\prod_{j \in G_{1}} l_{j}^{\rho} \prod_{j \in G_{2}}\left(k_{j}-1\right)^{\rho} \prod_{j \in G_{3}} k_{j}^{\rho}\right) \prod_{j=1}^{d} \cos k_{j} x_{j},
$$

где $G$ - разбиение множества $\{1, \ldots, d\}$ на непересекающиеся множества $G_{1}$, $G_{2}$ и $G_{3}$, а

$$
l_{j}= \begin{cases}k_{j}-1, & k_{j} \text { нечетное } \\ k_{j}, & k_{j} \text { четное. }\end{cases}
$$

Аналогично доказательству леммы 2.3 из [1; гл. 1] приходим к неравенству

$$
E_{\Gamma(N, \Omega)}^{\perp}(U(G, d, x))_{p} \ll\left\|\bar{U}_{N}^{\rho}\right\|_{p}+E^{(d-1)}(N, p),
$$

где $E^{(d-1)}(N, p)$ обозначает величину $E(N, p)$, соответствующую размерности $d-1$.

Используя леммы 2.1 и 2.2 и неравенство (6), получаем (5). Лемма доказана.

ЛЕмма 2.4. При $1<p \leqslant 2, \rho>\frac{1}{p}-1$ верно неравенство

$$
E_{\Gamma(N, \Omega)}^{\perp}\left(U_{N}^{\rho}\right)_{p} \ll E(N, p),
$$

где $E(N, p)$ - величина, определенная в лемме 2.2 .

ДокАЗАтЕЛЬСтво. Пусть

$$
2^{n-1} \leqslant N<2^{n}, \quad M=2^{l} N_{1}, \quad N_{1}=2^{r d+\sum_{j=1}^{d}\left|b_{j}\right|} \cdot N .
$$

Возьмем приближающую функцию

$$
g(x)=U_{N}^{\rho}(x, \alpha)-S_{Q\left(N_{1}\right)}\left(U_{M}^{\rho}(x, \alpha)\right),
$$

где $S_{Q\left(N_{1}\right)}\left(U_{M}^{\rho}(x, \alpha)\right)$ - частная сумма Фурье функции $U_{M}^{\rho}(x, \alpha)$, соответствующая множеству $Q\left(N_{1}\right)$.

Тогда из многомерной теоремы Литтлвуда-Пэли с учетом $\rho+1-\frac{1}{p}>0$ получаем

$$
\begin{aligned}
& \left\|U_{N}^{\rho}(x, \alpha)-g(x)\right\|_{p}^{p}=\left\|S_{Q\left(N_{1}\right)}\left(U_{M}^{\rho}(x, \alpha)\right)\right\|_{p}^{p} \\
& \quad \ll \sum_{s \in \varkappa\left(N_{1}\right)}\left\|\delta_{s}\left(U_{M}^{\rho}\right)\right\|_{p}^{p} \ll \sum_{s \in \varkappa\left(N_{1}\right)} 2^{p\left(\rho+1-\frac{1}{p}\right)\|s\|_{1}} \\
& \quad \ll \sum_{s \in \theta\left(N_{1}\right)} 2^{p\left(\rho+1-\frac{1}{p}\right)\|s\|_{1}} \asymp N_{1}^{\frac{p}{r}\left(\rho+1-\frac{1}{p}\right)} \sum_{s \in \theta\left(N_{1}\right)} \prod_{j=1}^{d} s_{j}^{-\frac{b_{j}}{r} p\left(\rho+1-\frac{1}{p}\right)} .
\end{aligned}
$$

Далее, применяя лемму 0.1 с $\gamma_{j}=\frac{b_{j}}{r}(p \rho+p-1)$, получаем лемму 2.4 .

При некоторых дополнительных условиях на $b_{j}$ мы можем указать точный порядок величин $E_{\Gamma(N, \Omega)}^{\perp}\left(U_{N}^{\rho}\right)_{p}$. 
Введем следующие обозначения $\left(p^{\prime}=\frac{p}{p-1}\right)$ :

$$
M_{1}=\max \left\{\frac{r}{p^{\prime}-1}, \frac{r}{\rho+1}, \frac{r}{p \rho+p-1}\right\}
$$

$m_{1}=\min \left\{\frac{r}{p^{\prime}-1}, \frac{r}{\rho+1}, \frac{r}{p \rho+p-1}\right\}, \quad m_{2}=\min \left\{\frac{r}{p \rho+p-1}, \frac{r}{3}, \frac{r}{\rho+2}\right\}$.

Лемма 2.5. Пусть $\rho>\frac{1}{p}$. Для $1<p \leqslant 2$ имеем:

1) $п р и b_{d}<m_{1}$

$$
E_{\Gamma(N, \Omega)}^{\perp}\left(U_{N}^{\rho}\right)_{p} \asymp N^{\frac{1}{r}\left(\rho+1-\frac{1}{p}\right)}(\log N)^{\frac{d-1}{p}-\frac{1}{r}\left(\rho+1-\frac{1}{p}\right)\left(b_{1}+\cdots+b_{d}\right)} ;
$$

2) при $b_{\nu}<m_{1} \leqslant M_{1}<b_{\nu+1}$

$$
E_{\Gamma(N, \Omega)}^{\perp}\left(U_{N}^{\rho}\right)_{p} \asymp N^{\frac{1}{r}\left(\rho+1-\frac{1}{p}\right)}(\log N)^{\frac{\nu-1}{p}-\frac{1}{r}\left(\rho+1-\frac{1}{p}\right)\left(b_{1}+\cdots+b_{\nu}\right)} ;
$$

3) при $b_{1} \geqslant M_{1}, b_{2}>M_{1}$

$$
E_{\Gamma(N, \Omega)}^{\perp}\left(U_{N}^{\rho}\right)_{p} \asymp N^{\frac{1}{r}\left(\rho+1-\frac{1}{p}\right)}(\log N)^{-\frac{b_{1}}{r}\left(\rho+1-\frac{1}{p}\right)} .
$$

При $2 \leqslant p<\infty$ получаем, что:

1) $п р и b_{d}<m_{2}$ верно (7);

2) при $b_{\nu}<m_{2}<r<b_{\nu+1}$ верно (8);

3) при $b_{1} \geqslant r, b_{2}>r$ верно (9).

При $p=\infty, b_{2}>$ r будет

$$
E_{\Gamma(N, \Omega)}^{\perp}\left(U_{N}^{\rho}\right)_{\infty} \asymp N^{\frac{\rho+1}{r}}(\log N)^{-\frac{b_{1}}{r}(\rho+1)} .
$$

ДокАЗАтЕльство. Оценки сверху следуют из лемм 2.3 и 2.4. Докажем оценки снизу.

Пусть $1<p \leqslant 2$. Тогда

$$
\left(U_{N}^{\rho}(x, \alpha), \bar{U}_{3^{-d} N}^{0}(x, \alpha)\right) \leqslant E_{\Gamma(N, \Omega)}^{\perp}\left(U_{N}^{\rho}\right)_{p} \cdot\left\|\bar{U}_{3^{-d} N}^{0}\right\|_{p^{\prime}} .
$$

По лемме 2.2 с $\rho=0$ получаем, что $\left\|\bar{U}_{3^{-d} N}^{0}\right\|_{p^{\prime}}$ по порядку не превосходит:

1) $N^{\frac{1}{r p}}(\log N)^{\frac{d-1}{p^{\prime}}-\frac{1}{r p}\left(b_{1}+\cdots+b_{d}\right)}$, если $b_{d}<\frac{r}{p^{\prime}-1}$;

2) $N^{\frac{1}{r p}}(\log N)^{\frac{\nu-1}{p^{\prime}}-\frac{1}{r p}\left(b_{1}+\cdots+b_{\nu}\right)}$, если $b_{\nu}<\frac{r}{p^{\prime}-1}<b_{\nu+1}$;

3) $N^{\frac{1}{r p}}(\log N)^{-\frac{b_{1}}{r p}}$, если $b_{2}>\frac{r}{p^{\prime}-1}$.

С другой стороны,

$$
\left(U_{N}^{\rho}(x, \alpha), \bar{U}_{3^{-d} N}^{0}\right) \geqslant \sum_{s \in \theta\left(3^{-d} N\right)} 2^{\left\|s_{1}\right\|(\rho+1)} \asymp N^{\frac{\rho+1}{r}} \sum_{s \in \theta\left(3^{-d} N\right)} \prod_{j=1}^{d} s_{j}^{-\frac{b_{j}}{r}(\rho+1)} .
$$

Далее, по лемме 0.1 получаем, что последняя величина по порядку равна:

1) $N^{\frac{\rho+1}{r}}(\log N)^{d-1-\frac{\rho+1}{r}\left(b_{1}+\cdots+b_{d}\right)}$, если $b_{d}<\frac{r}{\rho+1}$; 
2) $N^{\frac{\rho+1}{r}}(\log N)^{\nu-1-\frac{\rho+1}{r}\left(b_{1}+\cdots+b_{\nu}\right)}$, если $b_{\nu}<\frac{r}{\rho+1}<b_{\nu+1}$;

3) $N^{\frac{\rho+1}{r}}(\log N)^{-\frac{b_{1}(\rho+1)}{r}}$, если $b_{2}>\frac{r}{\rho+1}$.

Соединяя эти оценки и учитывая условия на $b_{j}$, получаем нужную оценку снизу для $1<p \leqslant 2$.

Пусть, далее, $2 \leqslant p<\infty$. Считаем, что $N$ - достаточно большое натуральное число. Возьмем функцию $V_{N}(x, \alpha)=K_{2^{-k l} N}^{1}(x, \alpha)$, где $K_{M}^{\rho}(x, \alpha)$ определено выше. Легко видеть, что $\left\|V_{N}\right\|_{1} \asymp|Q(N)|$.

Далее,

$$
\left\|V_{N}\right\|_{2}^{2} \asymp \sum_{\nu=1}^{\left[\frac{1}{l} \log N\right]} \sum_{s \in \theta\left(\frac{N}{2^{l \nu}}\right)} \sum_{k \in \rho(s)} 2^{2\|s\|_{1}} \asymp \sum_{s \in \theta(N)} 2^{3\|s\|_{1}} \asymp N^{\frac{3}{r}} \sum_{s \in \theta(N)} \prod_{j=1}^{d} s_{j}^{-\frac{3 b_{j}}{r}} .
$$

По лемме 0.1 эта величина по порядку равна:

1) $N^{\frac{3}{r}}(\log N)^{d-1-\frac{3\left(b_{1}+\cdots+b_{d}\right)}{r}}$, если $b_{d}<\frac{r}{3}$;

2) $N^{\frac{3}{r}}(\log N)^{\nu-1-\frac{3\left(b_{1}+\cdots+b_{\nu}\right)}{r}}$, если $b_{\nu}<\frac{r}{3}<b_{\nu+1}$;

3) $N^{\frac{3}{r}}(\log N)^{-\frac{3 b_{1}}{r}}$, если $b_{2}>\frac{r}{3}$.

Интерполируя, для $1<q \leqslant 2$ получим

$$
\left\|V_{N}\right\|_{q} \leqslant\left\|V_{N}\right\|_{1}^{\frac{2-q}{q}} \cdot\left\|V_{N}\right\|_{2}^{\frac{2(q-1)}{q}} .
$$

Подставляя сюда $\left\|V_{N}\right\|_{1}$ и $\left\|V_{N}\right\|_{2}$, получаем оценку для $\left\|V_{N}\right\|_{q}$.

Теперь рассмотрим скалярное произведение

$$
\left(U_{N_{1}}^{\rho}, V_{N}\right), \quad \text { где } N_{1}=2^{r d+\sum_{j=1}^{d}\left|b_{j}\right|} N .
$$

С одной стороны, для $2 \leqslant p<\infty$ имеем

$$
\left|\left(U_{N_{1}}^{\rho}, V_{N}\right)\right| \leqslant E_{\Gamma(N, \Omega)}^{\perp}\left(U_{N_{1}}^{\rho}\right)_{p} \cdot\left\|V_{N}\right\|_{p^{\prime}} .
$$

С другой стороны,

$$
\left|\left(U_{N_{1}}^{\rho}, V_{N}\right)\right| \gg \sum_{s \in \theta(N)} 2^{\|s\|_{1}(\rho+2)} \asymp N^{\frac{\rho+2}{r}} \sum_{s \in \theta(N)} \prod_{j=1}^{d} s_{j}^{-\frac{b_{j}(\rho+2)}{r}} .
$$

По лемме 0.1 эта величина по порядку равна:

1) $N^{\frac{\rho+2}{r}}(\log N)^{d-1-\frac{(\rho+2)\left(b_{1}+\cdots+b_{d}\right)}{r}}$, если $b_{d}<\frac{r}{\rho+2}$;

2) $N^{\frac{\rho+2}{r}}(\log N)^{\nu-1-\frac{(\rho+2)\left(b_{1}+\cdots+b_{\nu}\right)}{r}}$, если $b_{\nu}<\frac{r}{\rho+2}<b_{\nu+1}$;

3) $N^{\frac{\rho+2}{r}}(\log N)^{-\frac{b_{1}(\rho+2)}{r}}$, если $b_{2}>\frac{r}{\rho+2}$.

Собирая воедино (10) и (11), получаем нужную оценку для $2 \leqslant p<\infty$.

Рассмотрим $p=\infty$. Аналогично предыдущему случаю с учетом $b_{2}>r$ получим, с одной стороны,

$$
\left|\left(U_{N_{1}}^{\rho}, V_{N}\right)\right| \gg N^{\frac{\rho+2}{r}}(\log N)^{-\frac{b_{1}(\rho+2)}{r}} .
$$


С другой стороны,

$$
\left|\left(U_{N_{1}}^{\rho}, V_{N}\right)\right| \leqslant E_{\Gamma(N, \Omega)}^{\perp}\left(U_{N_{1}}^{\rho}\right)_{\infty} \cdot N^{\frac{1}{r}}(\log N)^{-\frac{b_{1}}{r}} .
$$

Отсюда получим оценку снизу для $p=\infty$. Лемма 2.5 доказана.

Перейдем к неравенствам Джексона-Никольского для тригонометрических полиномов $t(x) \in T(\Gamma(N, \Omega))$.

Обозначим

$$
m_{3}=\min \left\{\frac{r\left(1-\frac{1}{p}\right)}{\rho+\frac{1}{p}}, \frac{r}{\rho+1}, \frac{r}{\rho-1}\right\}, \quad M_{3}=\max \left\{\frac{r\left(1-\frac{1}{p}\right)}{\rho+\frac{1}{p}}, \frac{r}{\rho+1}, \frac{r}{\rho-1}\right\} .
$$

ТЕОрема 2.1. Для величины

$$
A(p)=\sup _{t \in T(\Gamma(N, \Omega))} \frac{\left\|t^{(\rho)}(x, \alpha)\right\|_{\infty}}{\|t\|_{p}}
$$

верно следующее:

1) при $\rho>-1$ будет $A(1) \asymp N^{\frac{\rho+1}{r}}(\log N)^{-\frac{b_{1}(\rho+1)}{r}}$;

2) при $1<p<\infty, \rho>-\frac{1}{p}$ величина $A(p)$ по порядку равна (см. замечание после доказательства теоремы 2.1)

$$
\begin{array}{lll}
N^{\frac{\rho+\frac{1}{p}}{r}}(\log N)^{(d-1)\left(1-\frac{1}{p}\right)-\frac{\left(\rho+\frac{1}{p}\right)\left(b_{1}+\cdots+b_{d}\right)}{r}} & \text { npu } & b_{d}<m_{3}, \\
N^{\frac{\rho+\frac{1}{p}}{r}}(\log N)^{(\nu-1)\left(1-\frac{1}{p}\right)-\frac{\left(\rho+\frac{1}{p}\right)\left(b_{1}+\cdots+b_{\nu}\right)}{r}} & \text { npu } & b_{\nu}<m_{3} \leqslant M_{3}<b_{\nu+1}, \\
N^{\frac{\rho+\frac{1}{p}}{r}}(\log N)^{\frac{-b_{1}\left(\rho+\frac{1}{p}\right)}{r}} & \text { npu } & b_{2}>M_{3} .
\end{array}
$$

ДокАзАтЕЛЬство. Докажем оценки сверху.

Так как $t^{(\rho)}(x, \alpha)=t(x) * U_{N}^{\rho}(x, \alpha)$, то

$$
\left\|t^{(\rho)}(x, \alpha)\right\|_{\infty} \leqslant\|t\|_{p} E_{\Gamma(N, \Omega)}^{\perp}\left(U_{N}^{\rho}\right)_{p^{\prime}},
$$

и по леммам 2.3 и 2.4 мы получаем оценки сверху.

Докажем оценки снизу.

При $p=1$ возьмем вектор

$$
\widetilde{s}=\left(\widetilde{s}_{1}, 1, \ldots, 1\right) \in \theta(N), \quad \widetilde{s}_{1} \asymp \log N .
$$

Рассмотрим функцию

$$
A_{\widetilde{s}}(x, \alpha)=\left(V_{2^{\widetilde{s}_{1}-1}}\left(x_{1}+\frac{\alpha_{1} \pi}{2}\right)-V_{2^{\widetilde{s}_{1}-2}}\left(x_{1}+\frac{\alpha_{1} \pi}{2}\right)\right) \prod_{j=2}^{d} \cos \left(x_{j}+\frac{\alpha_{j} \pi}{2}\right)
$$


$V_{m}(\tau)$ - одномерное ядро Валле Пуссена. Тогда $\left\|A_{\widetilde{s}}(x, \alpha)\right\|_{1} \ll 1$. И, кроме того,

$$
\left|A_{\widetilde{s}}^{\rho}(0, \alpha)\right| \asymp 2^{\|\widetilde{s}\|_{1}(\rho+1)} \asymp N^{\frac{\rho+1}{r}}(\log N)^{\frac{-b_{1}(\rho+1)}{r}} .
$$

Отсюда следует оценка снизу при $p=1$.

Пусть теперь $1<p<\infty$.

Рассмотрим функцию $t(x)=S_{Q(N)}\left(U_{N}^{0}(x, \alpha)\right)$, где $S_{Q(N)}$ - частная сумма Фурье, соответствующая множеству $Q(N)$. Как отмечено выше, $Q(N) \subset \Gamma(N, \Omega)$. Оценка сверху $\|t\|_{p}$ следует из доказательства леммы 2.4 с $\rho=0,1<p \leqslant 2$ и из леммы 2.2 с $\rho=0,2 \leqslant p<\infty$. Оценим снизу $t^{(\rho)}(0, \alpha)$ :

$$
t^{(\rho)}(0, \alpha) \asymp \sum_{\nu=1}^{\left[\frac{1}{l} \log N\right]} \sum_{s \in \theta\left(\frac{N}{2^{l \nu}}\right)} 2^{\|s\|_{1}(\rho+1)} \asymp \sum_{s \in \theta(N)} N^{\frac{\rho+1}{r}} \prod_{j=1}^{d} s_{j}^{-\frac{b_{j}(\rho+1)}{r}} .
$$

Далее применение леммы 0.1 дает оценку снизу. Теорема доказана.

ЗАмечАние 2.1. Отметим, что для $1<p<\infty$ при рассмотрении оценок сверху и снизу важно сравнение чисел

$$
\frac{r\left(1-\frac{1}{p}\right)}{\rho+\frac{1}{p}}, \quad \frac{r}{\rho+1}, \quad \frac{r}{p-1} .
$$

При $\rho=p-2$ все эти числа равны. При $\rho>p-2$ будет

$$
\frac{r\left(1-\frac{1}{p}\right)}{\rho+\frac{1}{p}}<\frac{r}{\rho+1}<\frac{r}{\rho-1},
$$

а при $\rho<p-2$ будет

$$
\frac{r\left(1-\frac{1}{p}\right)}{\rho+\frac{1}{p}}>\frac{r}{\rho+1}>\frac{r}{p-1} .
$$

Поэтому в случае $1<p<\infty, \rho=p-2$ утверждение теоремы 2.1 имеет наиболее завершенный вид, т.е. $A(p)$ по порядку равно:

1) $N^{\frac{p-2+\frac{1}{p}}{r}}(\log N)^{(d-1)\left(1-\frac{1}{p}\right)-\frac{\left(p-2+\frac{1}{p}\right)\left(b_{1}+\cdots+b_{d}\right)}{r}}$, если $b_{d}<\frac{r}{p-1}$;

2) $N^{\frac{p-2+\frac{1}{p}}{r}}(\log N)^{(\nu-1)\left(1-\frac{1}{p}\right)-\left(p-2+\frac{1}{p}\right) \frac{\left(b_{1}+\cdots+b_{\nu}\right)}{r}}(\log \log N)^{\mu\left(1-\frac{1}{p}\right)}, \quad$ если $\quad b_{\nu}<$ $\frac{r}{p-1}=b_{\nu+1}=\cdots=b_{\nu+\mu}<b_{\nu+\mu+1}$;

3) $N^{\frac{p-2+\frac{1}{p}}{r}}(\log N)^{-\left(1-\frac{1}{p}\right)}(\log \log N)^{(\nu-1)\left(1-\frac{1}{p}\right)}$, если $\frac{r}{p-1}=b_{1}=\cdots=b_{\nu}<$ $b_{\nu+1}$

4) $N^{\frac{p-2+\frac{1}{p}}{r}}(\log N)^{\frac{-b_{1}\left(p-2+\frac{1}{p}\right)}{r}}$, если $b_{2}>\frac{r}{p-1}$.

СлеДСтвиЕ 2.1. Пусть

$$
V_{N, \Omega}(x)=\sum_{s \in Q(N)} A_{s}^{0}(x, 0) .
$$

Норма $\left\|V_{N, \Omega}(x)\right\|_{1}$ по порядку не менъше, чем: 
1) $(\log N)^{d-1-\frac{b_{2}+\cdots+b_{d}}{r}}$, ecлu $b_{d}<r$;

2) $(\log N)^{\nu-1-\frac{b_{2}+\cdots+b_{\nu}}{r}}(\log \log N)^{\mu}$, если $b_{\nu}<r=b_{\nu+1}=\cdots=b_{\nu+\mu}<$ $b_{\nu+\mu+1}$

3) $(\log \log N)^{\nu-1}$, если $r=b_{1}=\cdots=b_{\nu}<b_{\nu+1}$.

ДокаЗАТЕЛЬСтво. Имеем $V_{N, \Omega}(0) \asymp|\Gamma(N, \Omega)|$. С другой стороны, по теореме 2.1 с $\rho=0$

$$
\left\|V_{N, \Omega}(x)\right\|_{\infty} \ll N^{\frac{1}{r}}(\log N)^{-\frac{b_{1}}{r}}\left\|V_{N, \Omega}(x)\right\|_{1} .
$$

Отсюда получаем утверждение следствия.

Это следствие показывает, что нормы $\left\|V_{N, \Omega}(x)\right\|_{1}$ не ограничены в совокупности. Как и в [1; теорема 1.2.2], отметим одно усиление теоремы 2.1 при $1<p \leqslant 2$.

TEOPEMA 2.2. Пусmъ

$$
1<p \leqslant 2, \quad t(x)=\sum_{k \in Q(N)} \widehat{t}(k) e^{i(k, x)} .
$$

Тогда $\sum_{k \in Q(N)}|\widehat{t}(k)|$ по порядку не превосходит:

1) $N^{\frac{1}{r p}}(\log N)^{(d-1)\left(1-\frac{1}{p}\right)-\frac{b_{1}+\cdots+b_{d}}{r p}}\|t\|_{p}$, если $b_{d}<\frac{r}{p^{\prime}-1}$;

2) $N^{\frac{1}{r p}}(\log N)^{(\nu-1)\left(1-\frac{1}{p}\right)-\frac{b_{1}+\cdots+b_{\nu}}{r p}}(\log \log N)^{\mu\left(1-\frac{1}{p}\right)}\|t\|_{p}, \quad$ ecлu $\quad b_{\nu}<\frac{r}{p^{\prime}-1}=$ $b_{\nu+1}=\cdots=b_{\nu+\mu}<b_{\nu+\mu+1}$

3) $N^{\frac{1}{r p}}(\log N)^{\frac{1}{p}-1}(\log \log N)^{(\nu-1)\left(1-\frac{1}{p}\right)}\|t\|_{p}$, ecлu $\frac{r}{p^{\prime}-1}=b_{1}=\cdots=b_{\nu}<b_{\nu+1}$;

4) $N^{\frac{1}{r p}}(\log N)^{-\frac{b_{1}}{r p}}\|t\|_{p}$, если $b_{2}>\frac{r}{p^{\prime}-1}$.

Причем константы в этих неравенствах не зависят от $N$, но зависят от $p$.

Доказательство проводится аналогично доказательству теоремы 1.2 .2 из [1] с применением леммы 0.1 с $\gamma_{j}=b_{j} \frac{p^{\prime}-1}{r}$.

Tеорема 2.3. Пусть $1 \leqslant q \leqslant p<\infty, p>1, \rho \geqslant 0$. Тогда

$$
\sup _{t \in T(\Gamma(N, \Omega))} \frac{\left\|t^{(\rho)}(x, \alpha)\right\|_{p}}{\|t\|_{q}} \asymp N^{\frac{\rho+\frac{1}{q}-\frac{1}{p}}{r}}(\log N)^{-\frac{b_{1}\left(\rho+\frac{1}{q}-\frac{1}{p}\right)}{r}} .
$$

Оценки сверху доказываются аналогично доказательству теоремы 1.2.3 из [1]. Для доказательства оценок снизу берем функцию $\left.A_{\widetilde{s}}(x, 0)\right)$ из доказательства теоремы 2.1 .

СлеДСТВИЕ 2.2. Пусть $p>1$. Тогда для произволъного полинома $t(x) \in$ $T(\Gamma(N, \Omega))$ сумма $\sum_{k \in \Gamma(N, \Omega)}|\widehat{t}(k)|$ по порядку не превосходит:

1) $N^{\frac{1}{r}}(\log N)^{\frac{d-1}{p^{\prime}}-\frac{b_{1}}{r}-\frac{b_{2}+\cdots+b_{d}}{r p}}\|t\|_{1}$, ecлu $b_{d}<\frac{r}{p^{\prime}-1}$;

2) $N^{\frac{1}{r}}(\log N)^{\frac{\nu-1}{p^{\prime}}-\frac{b_{1}}{p}-\frac{b_{2}+\cdots+b_{\nu}}{r p}}(\log \log N)^{\frac{\mu}{p^{\prime}}}\|t\|_{1}$, ecлu $b_{\nu}<\frac{r}{p^{\prime}-1}=b_{\nu+1}=\cdots=$ $b_{\nu+\mu}<b_{\nu+\mu+1}$; 
3) $N^{\frac{1}{r}}(\log N)^{\left(\frac{b_{1}}{r}+1\right)\left(\frac{1}{p}-1\right)}(\log \log N)^{\frac{\nu-1}{p^{\prime}}}\|t\|_{1}$, ecлu $\frac{r}{p^{\prime}-1}=b_{1}=\cdots=b_{\nu}<b_{\nu+1}$;

4) $N^{\frac{1}{r}}(\log N)^{-\frac{b_{1}}{r}}\|t\|_{1}$, если $b_{2}>\frac{r}{p^{\prime}-1}$.

Причем константы в неравенствах не зависят от $N$, но зависят от $p$.

Доказательство следствия аналогично доказательству следствия из теоремы 1.2.3 из [1] с дополнительным применением леммы 0.1 .

\section{§ 3. Линейные приближения}

В [1; гл. 2, п. 5] для классов Никольского и Соболева изучалась возможность приближения функций линейными операторами, которые дают приближения порядка наилучшего. Мы рассмотрим аналогичную задачу для классов $H_{q}^{\Omega}$, обобщающих классы Никольского.

Через $E_{Q(N)}\left(H_{q}^{\Omega}\right)_{p}$ обозначим наилучшее приближение класса $H_{q}^{\Omega}$ в норме $L_{p}$ тригонометрическими полиномами из $T(Q(N))$, т.е.

$$
E_{Q(N)}\left(H_{q}^{\Omega}\right)_{p}=\sup _{f \in H_{q}^{\Omega}} E_{Q(N)}(f)_{p}, \quad E_{Q(N)}(f)_{p}=\inf _{t \in T(Q(N))}\|f-t\|_{p} .
$$

Рассмотрим линейные ограниченные операторы $\mathcal{L}_{N}$ такие, что

$$
\left\|f-\mathcal{L}_{N} f\right\|_{\infty} \ll E_{Q(N)}\left(H_{\infty}^{\Omega}\right)_{\infty} .
$$

Из результатов работы [3] следует, что

$$
E_{Q(N)}\left(H_{\infty}^{\Omega}\right)_{\infty} \ll \frac{1}{N}(\log N)^{d-1} .
$$

Итак, считаем, что для $f \in H_{\infty}^{\Omega}$ будет

$$
\left\|f-\mathcal{L}_{N} f\right\|_{\infty} \ll \frac{1}{N}(\log N)^{d-1} .
$$

Как отмечено выше, можно брать $\Gamma(N, \Omega)$ вместо $Q(N)$.

ТеОрема 3.1. Пусть линейные ограниченные операторы $\mathcal{L}_{N}$ ставят в соответствие функиии $f(x) \in H_{\infty}^{\Omega}$ тригонометрический полином $t(x)$, принадлежащий $T(\Gamma(N, \Omega))$, так, что

$$
\left\|f-\mathcal{L}_{N} f\right\|_{\infty} \ll \frac{1}{N}(\log N)^{d-1} .
$$

Тогда:

1) если $b_{1} \leqslant \cdots \leqslant b_{\nu}<0=b_{\nu+1}=\cdots=b_{\nu+\mu}<b_{\nu+\mu+1}, b_{d}<r \delta, b_{1}>-\alpha r$, где $\delta>0$ - достаточно малое число, $0<\alpha<\frac{d-1-\delta^{2}(d-\nu-\mu)}{p+\nu-1}$, причем $p=1+\delta$, то для нормы оператора $\mathcal{L}_{N}$ верно неравенство

$$
\left\|\mathcal{L}_{N}\right\| \gg(\log N)^{\beta},
$$

где $0<\beta=\frac{d-1-\alpha(p+\nu-1)-\delta^{2}(d-\nu-\mu)}{p}$;

2) если $b_{1}>0$, mo

$$
\left\|\mathcal{L}_{N}\right\| \gg(\log N)^{d-1-\frac{b_{2}+\cdots+b_{d}}{r}} .
$$


ЗАмечАниЕ. Так как в условиях теоремы $3.1 d-1-\frac{b_{2}+\cdots+b_{d}}{r}>0$, то нормы $\left\|\mathcal{L}_{N}\right\|$ не ограничены при $N \rightarrow+\infty$.

ДоКАЗАТЕЛЬСтво тЕоремы 3.1. Как сказано в [1] при доказательстве теоремы 2.5.1, в качестве $\mathcal{L}_{N}$ можно брать оператор свертки с ядром

$$
L_{N}=\sum_{k \in \Gamma(N, \Omega)} c_{N, k} e^{i(k, x)}
$$

Пусть $b_{1} \leqslant \cdots \leqslant b_{\nu}<0=b_{\nu+1}=\cdots=b_{\nu+\mu}<b_{\nu+\mu+1}$. Тогда легко видеть, что если

$$
\prod_{j=1}^{d}\left|k_{j}\right| \leqslant c \cdot N^{\frac{1}{r}}(\log N)^{-\frac{\left(b_{\nu+\mu+1}+\cdots+b_{d}\right)}{r}},
$$

то $k=\left(k_{1}, \ldots, k_{d}\right) \in \Gamma(N, \Omega)$, т.е. $\Gamma\left(N_{2}\right) \subset \Gamma(N, \Omega)$, где

$$
\Gamma\left(N_{2}\right)=\left\{k=\left(k_{1}, \ldots, k_{d}\right):\left|k_{j}\right| \in \mathbb{N}, j=1, \ldots, d, \prod_{j=1}^{d}\left|k_{j}\right| \leqslant N_{2}\right\}
$$

- гиперболический крест,

$$
N_{2}=c \cdot N^{\frac{1}{r}}(\log N)^{-\frac{\left(b_{\nu+\mu+1}+\cdots+b_{d}\right)}{r}} .
$$

Если теперь взять функцию $f(x) \in H_{\infty}^{\Omega}$ со спектром из $\Gamma\left(N_{2}\right)$, то получим, что

$$
\mathcal{L}_{N}(f)=f * \sum_{k \in \Gamma\left(N_{2}\right)} c_{N, k} e^{i(k, x)} .
$$

Обозначим через $\mathcal{L}_{N}^{(1)}(f)$ оператор свертки с ядром

$$
L_{N}^{(1)}=\sum_{k \in \Gamma\left(N_{2}\right)} c_{N, k} e^{i(k, x)} .
$$

Тогда для любой функции $f(x) \in H_{\infty}^{\Omega}$ со спектром из $\Gamma\left(N_{2}\right)$ будет

$$
\left\|f-\mathcal{L}_{N}^{(1)}(f)\right\|_{\infty} \ll \frac{1}{N}(\log N)^{d-1} .
$$

Для дальнейшего доказательства нам понадобится следующая

Лемма 3.1. Существует $\varepsilon>0$ такое, что для всех натуралъных $N$ будет

$$
\sum_{k \in \Gamma(N, \Omega)}\left|c_{N, k}\right| \geqslant \varepsilon \cdot N^{\frac{1}{r}}(\log N)^{d-1-\frac{b_{\nu+\mu+1}+\cdots+b_{d}}{r}} .
$$

ДокАЗАТЕЛьство. Используем указанный выше оператор $\mathcal{L}_{N}^{(1)}$ для приближения функции $f(x) \in H_{\infty}^{\Omega}$ со спектром из $\Gamma\left(N_{2}\right)$. 
Предполагая противное, а именно, что $\sum_{k \in \Gamma\left(N_{2}\right)}\left|c_{N, k}\right|<\varepsilon\left|\Gamma\left(N_{2}\right)\right|$, как и в [1] при доказательстве леммы 2.5.1, получим для числа элементов множества

$$
\begin{gathered}
Q=\left\{s=\left(s_{1}, \ldots, s_{d}\right): s_{j} \in \mathbb{N}, j=1, \ldots, d, \rho(\mathrm{s}) \subset \Gamma\left(N_{2}\right),\right. \\
\left.\exists k^{s} \in \rho(s):\left|c_{N, k^{s}}\right| \leqslant \frac{1}{2}\right\}
\end{gathered}
$$

следующую оценку снизу:

$$
|Q| \gg\left(\log N_{2}\right)^{d-1} \log \frac{1}{\varepsilon} \gg(\log N)^{d-1} \log \frac{1}{\varepsilon}, \quad \varepsilon>(\log N)^{1-d} .
$$

Далее, рассмотрим функцию

$$
f(x)=\sum_{s \in Q}\left(\prod_{j=1}^{d} 2^{r s_{j}} s_{j}^{b_{j}}\right)^{-1} e^{i\left(k^{s}, x\right)} .
$$

Напомним, что $\left|c_{N, k^{s}}\right| \leqslant \frac{1}{2}$. По теореме $0.2 f \in H_{\infty}^{\Omega}$. Имеем

$$
\left|f(0)-\left(\mathcal{L}^{(1)} f\right)(0)\right|=\left|\sum_{s \in Q}\left(1-c_{N, k^{s}}\right)\left(\prod_{j=1}^{d} 2^{r s_{j}} s_{j}^{b_{j}}\right)^{-1}\right| \gg \frac{|Q|}{N} .
$$

Отсюда с учетом (13) и (14) получаем, что $\log \frac{1}{\varepsilon} \leqslant c$. Полученное противоречие доказывает лемму.

Продолжим доказательство теоремы 3.1.

Сначала рассмотрим случай

$$
b_{1} \leqslant \cdots \leqslant b_{\nu}<0=b_{\nu+1}=\cdots=b_{\nu+\mu}<b_{\nu+\mu+1} .
$$

Учитывая условия на $b_{j}$ из леммы 3.1 и следствия 2.2 , получим

$$
\left\|L_{N}\right\|_{1} \gg \frac{(\log N)^{d-1-\frac{b_{\nu+\mu+1}+\cdots+b_{d}}{r}}}{(\log N)^{\frac{d-1}{p^{\prime}}-\frac{b_{1}}{r}-\frac{b_{2}+\cdots+b_{d}}{r p}}} .
$$

Далее, взяв $p=1+\delta$, где $\delta>0$ достаточно малое, получаем утверждение теоремы 3.1 в случае 1$)$. Взяв $b_{1}>0$, можно выбрать $p>1, p$ близко к 1 , так, чтобы было $b_{1}>\frac{r}{p^{\prime}-1}$, и аналогично предыдущему случаю получаем утверждение теоремы 3.1 в случае 2). Теорема 3.1 доказана.

Если некоторые $b_{j}$ больше $r$, т.е. $b_{\nu} \leqslant r<b_{\nu+1}$, то возникает эффект "не своих" гиперболических крестов. В этом случае наряду с $Q(N)$ рассмотрим множества

$$
Q^{\prime}(N)=\bigcup_{s \in \varkappa^{\prime}(N)} \rho(s)
$$

где

$$
\varkappa^{\prime}(N)=\left\{s=\left(s_{1}, \ldots, s_{d}\right): s_{j} \in \mathbb{N}, j=1, \ldots, d, \prod_{j=1}^{d} 2^{r s_{j}} s_{j}^{b_{j}^{\prime}} \leqslant N\right\}
$$


причем $b_{j}^{\prime}=b_{j}$ при $j=1, \ldots, \nu, r<b_{j}^{\prime}<b_{j}$ при $j=\nu+1, \ldots, d$. В этом случае по теореме 0.1

$$
|Q(N)| \asymp\left|Q^{\prime}(N)\right|,
$$

но приближения $E_{Q^{\prime}(N)}\left(H_{\infty}^{\Omega}\right)_{\infty}$ по порядку меньше, чем $E_{Q(N)}\left(H_{\infty}^{\Omega}\right)_{\infty}$. Этот эффект для классов Никольского установил С. А. Теляковский (см. [8]).

Автором доказано, что если $b_{\nu} \leqslant r<b_{\nu+1}, b_{j}-b_{j}^{\prime}>1, j=\nu+1, \ldots, d$, то

$$
E_{Q^{\prime}(N)}\left(H_{\infty}^{\Omega}\right)_{\infty} \ll \frac{1}{N}(\log N)^{\nu-1}
$$

(формулировку этого утверждения см. в [4]).

Теорема 3.2. Пусть $b_{\nu} \leqslant r<b_{\nu+1}, \nu>1, b_{\nu+1}-r>1$. Пусть последовательность линейных ограниченных операторов $\mathcal{L}_{N}$ ставит в соответствие функции $f(x)$ тригонометрический полином со спектром из $Q^{\prime}(N)$, причем $b_{j}^{\prime}=b_{j}$ при $j=1, \ldots, \nu, r<b_{j}^{\prime}<b_{j}$ при $j=\nu+1, \ldots, d u$, кроме того, $b_{j}-b_{j}^{\prime}>1$ при $j=\nu+1, \ldots, d$, и для всякой функиии $f(x) \in H_{\infty}^{\Omega}$ верно неравенство

$$
\left\|f-\mathcal{L}_{N}(f)\right\|_{\infty} \ll \frac{1}{N}(\log N)^{\nu-1} .
$$

Тогда:

1) если $b_{1} \leqslant \cdots \leqslant b_{\xi}<0=b_{\xi+1}=\cdots=b_{\xi+\zeta}<b_{\xi+\zeta+1} \leqslant \cdots \leqslant b_{\nu}<r \delta<r<$ $b_{\nu+1}, p=1+\delta, b_{1}>-\alpha r$, где $0<\alpha<\frac{\nu-1-\delta^{2}(\nu-\xi-\zeta)}{p+\xi-1}$, mo

$$
\left\|\mathcal{L}_{N}\right\| \gg(\log N)^{\beta}
$$

где $0<\beta=\frac{\nu-1-\alpha(p+\xi-1)-\delta^{2}(\nu-\xi-\zeta)}{p}$;

2) если $b_{1}>0$, mo

$$
\left\|\mathcal{L}_{N}\right\| \gg(\log N)^{\nu-1-\frac{b_{2}+\cdots+b_{\nu}}{r}} .
$$

ДокАЗАТЕЛЬСтво. В условиях теоремы

$$
E_{Q^{\prime}(N)}\left(H_{\infty}^{\Omega}\right)_{\infty} \ll \frac{1}{N}(\log N)^{\nu-1}
$$

Как и в теореме 3.1 , достаточно рассмотреть оператор свертки с ядром

$$
L_{N}(x)=\sum_{s \in \varkappa^{\prime}(N)} \sum_{k \in \rho(s)} c_{N, k} e^{i(k, x)} .
$$

Для любой функции $f(x) \in H_{\infty}^{\Omega}$ со спектром из множества

$$
Q^{(\nu)}(N)=\bigcup_{s \in \varkappa^{(\nu)}(N)} \rho(s),
$$

где

$$
\begin{gathered}
\varkappa^{(\nu)}(N)=\left\{s=\left(s_{1}, \ldots, s_{d}\right): s_{j} \in \mathbb{N}, j=1, \ldots, d, \prod_{j=1}^{\nu} 2^{r s_{j}} s_{j}^{b_{j}^{\prime}} \leqslant N,\right. \\
\left.s_{j}=1, j=\nu+1, \ldots, d\right\},
\end{gathered}
$$

будет $\left\|f-\mathcal{L}_{N}(f)\right\|_{\infty} \ll \frac{1}{N}(\log N)^{\nu-1}$. 
Предположим, что $b_{1} \leqslant \cdots \leqslant b_{\xi}<0=b_{\xi+1}=\cdots=b_{\xi+\zeta}<b_{\xi+\zeta+1}$. Отсюда, как и в теореме 3.1 , следует, что

$$
\sum_{k \in Q^{(\nu)}(N)}\left|c_{N, k}\right| \gg \varepsilon \cdot N^{\frac{1}{r}}(\log N)^{\nu-1-\frac{b_{\xi+\zeta+1}+\cdots+b_{\nu}}{r}} .
$$

Следовательно,

$$
\sum_{k \in Q^{\prime}(N)}\left|c_{N, k}\right| \gg \varepsilon \cdot N^{\frac{1}{r}}(\log N)^{\nu-1-\frac{b_{\xi+\zeta+1}+\cdots+b_{\nu}}{r}} .
$$

И далее так же, как и в теореме 3.1 , мы получаем оценки снизу для $\left\|\mathcal{L}_{N}\right\|$. Теорема 3.2 доказана.

Перейдем к рассмотрению метрики $L_{1}$. Из результатов работы [3] следует, что

$$
E_{Q(N)}\left(H_{1}^{\Omega}\right)_{1} \ll \frac{1}{N}(\log N)^{d-1} .
$$

Автором доказано, что если $b_{\nu} \leqslant r<b_{\nu+1}, b_{j}-b_{j}^{\prime}>1, j=\nu+1, \ldots, d$, то

$$
E_{Q^{\prime}(N)}\left(H_{1}^{\Omega}\right)_{1} \ll \frac{1}{N}(\log N)^{\nu-1}
$$

(формулировку см. в [4]).

TeOpema 3.3. Пусms

$$
\begin{aligned}
b_{1} \leqslant \cdots \leqslant b_{\xi}<0 & =b_{\xi+1}=\cdots=b_{\xi+\eta}<b_{\xi+\eta+1} \leqslant \cdots \leqslant b_{\nu}<r \\
& =b_{\nu+1}=\cdots=b_{\nu+\mu}<b_{\nu+\mu+1}
\end{aligned}
$$

Пусть также

$$
r>\frac{d+b_{\mu+1}+\cdots+b_{d}}{d-1} .
$$

Предположим, что линейный ограниченный оператор $\mathcal{L}_{N}$ cтавит в соответствие каждой функиии $f(x) \in H_{1}^{\Omega}$ тригонометрический полином со спектром из $Q(N)$ maк, что

$$
\left\|f-\mathcal{L}_{N}(f)\right\|_{1} \ll \frac{1}{N}(\log N)^{d-1} .
$$

Тогда существует число а>0 такое, что

$$
\left\|\mathcal{L}_{N}\right\| \geqslant c_{a}(\log N)^{a}
$$

где с са зависит от а и не зависит от $N$.

ДоказАтельство. Пусть задано $N$. Рассмотрим функцию

$$
f_{N}(x)=\sum_{s \in \varkappa(N)} \Omega\left(2^{-s}\right) \prod_{j=1}^{d} s_{j}^{-1} \cdot \delta_{s}(x),
$$


где

$$
\delta_{s}(x)=\sum_{s \in \rho(s)} e^{i(k, x)} .
$$

Как легко видеть, по теореме 0.2 функция $B \cdot f_{N}(x)$ принадлежит $H_{1}^{\Omega}$ при некотором $B>0$. Имеем

$$
\left\|f_{N}-\mathcal{L}_{N}\left(f_{N}\right)\right\|_{1} \ll \frac{1}{N}(\log N)^{d-1} .
$$

Как уже отмечалось, достаточно рассмотреть оператор свертки с ядром

$$
L_{N}(x)=\sum_{k \in Q(N)} c_{N, k} e^{i(k, x)} .
$$

По теореме 2.3 с $\rho=0$ с учетом (15) получим $(q>1)$

$$
\left\|f_{N}-\mathcal{L}_{N}\left(f_{N}\right)\right\|_{q} \ll N^{\frac{1-\frac{1}{q}-r}{r}}(\log N)^{d-1-\frac{b_{1}\left(1-\frac{1}{q}\right)}{r}} .
$$

Будем считать, что $q>1, q$ близко к 1 . Обозначим $1-\frac{1}{q}=\delta$, т.е. $q=\frac{1}{1-\delta}$. По лемме 0.3 с учетом (16) получим

$$
\begin{aligned}
J & \equiv\left(\sum_{s}\left(\sum_{k \in \rho(s)}\left|\widehat{f}_{N}(k)\right|^{2}\left|1-c_{N, k}\right|^{2}\right)^{\frac{q}{2}} 2^{\|s\|_{1}\left(\frac{q}{2}-1\right)}\right)^{\frac{1}{q}} \\
& \ll N^{\frac{1-\frac{1}{q}-r}{r}}(\log N)^{d-1-\frac{b_{1}\left(1-\frac{1}{q}\right)}{r}} .
\end{aligned}
$$

Предположим, как и в лемме 3.1 , противное, т.е. для $\varepsilon>0$ будет

$$
\sum_{k \in Q(N)}\left|c_{N, k}\right|<\varepsilon|Q(N)| .
$$

Пусть

$$
V=\left\{k \in Q(N):\left|c_{N, k}\right|>\frac{1}{2}\right\} .
$$

Тогда из неравенства (18) следует, что $|V|<2 \varepsilon|Q(N)|$.

Положим $V_{s}=V \cap \rho(s)$. Тогда $\sum_{s}\left|V_{s}\right|<2 \varepsilon|Q(N)|$ и для $J$ при указанных $q$ получаем

$$
\begin{aligned}
J^{q} & \gg \sum_{s}\left(\sum_{\substack{k \in \rho(s) \\
k \notin V}}\left|\widehat{f}_{N}(k)\right|^{2}\right)^{\frac{q}{2}} 2^{\|s\|_{1}\left(\frac{q}{2}-1\right)} \\
& \gg \sum_{s}\left(1-\frac{\left|V_{s}\right|}{|\rho(s)|}\right) 2^{\|s\|_{1} \frac{q}{2}}\left(\Omega\left(2^{-s}\right)\right)^{q} \prod_{j=1}^{d} s_{j}^{-q} 2^{\|s\|_{1}\left(\frac{q}{2}-1\right)} \\
& =\sum_{s}\left(1-\frac{\left|V_{s}\right|}{|\rho(s)|}\right) 2^{\|s\|_{1}(q-1-r q)} \prod_{j=1}^{d} s_{j}^{-q\left(b_{j}+1\right)}
\end{aligned}
$$


Поскольку $q$ близко к 1 , то $q-1-r q<0$. Имеем

$$
J^{q} \gg \sum_{\|s\|_{1}>m} 2^{\|s\|_{1}(q-1-r q)} \prod_{j=1}^{d} s_{j}^{-q\left(b_{j}+1\right)},
$$

где $m$ выбрано максимально возможным таким, что для множества $Q_{m}=$ $\bigcup_{\|s\|_{1} \leqslant m} \rho(s)$ выполнено неравенство $\left|Q_{m}\right|<2 \varepsilon|Q(N)|$.

Поскольку $\left|Q_{m}\right| \asymp 2^{m} m^{d-1}$, то, полагая $|Q(N)|=N^{\frac{1}{r}} \Phi(N)$ (см. оценку количества элементов множества $Q(N)$ - теорему 0.1 ), продолжаем оценку снизу:

$$
\begin{aligned}
J^{q} & \gg 2^{m(q-1-r q)} m^{d-1-q d-q\left(b_{1}+\cdots+b_{d}\right)} \\
& \asymp\left(\varepsilon N^{\frac{1}{r}} \frac{\Phi(N)}{(\log N)^{d-1}}\right)^{q-1-r q}(\log N)^{d-1-q d-q\left(b_{1}+\cdots+b_{d}\right)} .
\end{aligned}
$$

Соединяя оценки (17) и (19), получим

$$
\varepsilon \gg\left(\left(\frac{\Phi(N)}{(\log N)^{d-1}}\right)^{1-\frac{1}{q}-r}(\log N)^{(d-1)\left(\frac{1}{q}-1\right)+\frac{b_{1}\left(1-\frac{1}{q}\right)}{r}-d-\left(b_{1}+\cdots+b_{d}\right)}\right)^{-\frac{q}{q-1-r q}} .
$$

Учитывая условия на $b_{j}$ и оценку $|Q(N)|$, получаем

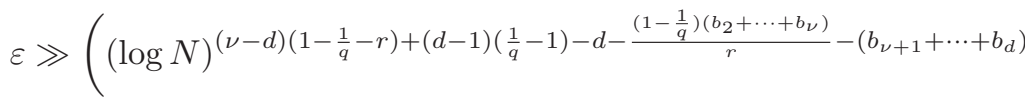

$$
\begin{aligned}
& \left.\times(\log \log N)^{\mu\left(1-\frac{1}{q}-r\right)}\right)^{\frac{1}{r-1+\frac{1}{q}}} .
\end{aligned}
$$

Учитывая, что $q=\frac{1}{1-\delta}$, получаем противоречие в (18).

Итак,

$$
\begin{aligned}
\sum_{k \in Q(N)}\left|c_{N, k}\right| \geqslant c_{\delta} & |Q(N)|(\log N)^{d-\nu-(d-1) \frac{\delta}{r-\delta}-\frac{d}{r-\delta}-\frac{\delta\left(b_{2}+\cdots+b_{\nu}\right)}{r(r-\delta)}-\frac{b_{\nu+1}+\cdots+b_{d}}{r-\delta}} \\
& \times(\log \log N)^{-\mu}
\end{aligned}
$$

Считаем, что $p>1, p$ близко к 1 , т.е. $1-\frac{1}{p}=\delta_{1}, p=\frac{1}{1-\delta_{1}}$. Кроме того, выбираем $p$ так, чтобы было

$$
b_{\xi+\eta}<\frac{r}{p^{\prime}-1}<b_{\xi+\eta+1} .
$$

Соединяя эту оценку со следствием 2.2 и теоремой 0.1 , получаем

$$
\left\|L_{N}\right\|_{1} \gg(\log N)^{a},
$$

где

$$
\begin{gathered}
a=d-1-\frac{1}{r} \delta_{1}\left(b_{2}+\cdots+b_{\xi+\eta}\right)-\frac{1}{r}\left(b_{\xi+\eta+1}+\cdots+b_{\nu}\right)-\delta_{1}(\xi+\eta-1) \\
-(d-1) \frac{\delta}{r-\delta}-\frac{d}{r-\delta}-\frac{\delta\left(b_{2}+\cdots+b_{\nu}\right)}{r(r-\delta)}-\frac{b_{\nu+1}+\cdots+b_{d}}{r-\delta} .
\end{gathered}
$$


Считая $\delta$ и $\delta_{1}$ достаточно малыми положительными числами и учитывая, что $r$ удовлетворяет условию теоремы, получим, что $a>0$.

Теорема 3.3 доказана.

Теорема 3.4. Пусть $b_{j}, j=1, \ldots, d$, удовлетворяют условиям теоремы 3.3. Пусть, кроме того,

$$
b_{\nu+1}-r>1, \quad r>\frac{\nu+b_{\mu+\xi+1}+\cdots+b_{d}}{d-1} .
$$

Предположим, что линейный ограниченный оператор $\mathcal{L}_{N}$ cтавит в соответствие функиии $f(x) \in H_{1}^{\Omega}$ тригонометрический полином со спектром из $Q^{\prime}(N)$ такой, что

$$
\left\|f-\mathcal{L}_{N} f\right\|_{1} \ll \frac{1}{N}(\log N)^{\nu-1} .
$$

Тогда $\left\|\mathcal{L}_{N}\right\| \geqslant c_{a}(\log N)^{a}$, где $a>0$.

ДокАЗАтельство. Аналогично доказательству теоремы 3.3, полагая 1 $\frac{1}{q}=\delta$, получаем

$$
\varepsilon \gg\left(\left(\frac{\Phi(N)}{(\log N)^{d-1}}\right)^{\delta-r}(\log N)^{\frac{d-1}{q}-(\nu-1)+\frac{b_{1} \delta}{r}-d-\left(b_{1}+\cdots+b_{d}\right)}\right)^{\frac{1}{r-\delta}} .
$$

Учитывая, что по теореме 0.1

$$
\Phi(N) \asymp(\log N)^{(\nu-1)-\frac{1}{2}\left(b_{1}+\cdots+b_{\nu}\right)}(\log \log N)^{\mu},
$$

получаем

$$
\varepsilon \gg(\log N)^{\frac{b}{r-\delta}}(\log \log N)^{-\mu},
$$

где

$b=(d-1)\left(r-\delta+\frac{1}{q}\right)-(\nu-1)(r-\delta+1)-\frac{\delta}{r}\left(b_{2}+\cdots+b_{\nu}\right)-\left(b_{\nu+1}+\cdots+b_{d}\right)-d$.

Следовательно,

$$
\sum_{k \in Q^{\prime}(N)}\left|c_{N, k}\right| \geqslant \mathrm{C}_{\delta}\left|Q^{\prime}(N)\right|(\log N)^{\frac{b}{r-\delta}}(\log \log N)^{-\mu} .
$$

Соединяя это неравенство со следствием 2.2 , для $L_{N}=\sum_{k \in Q^{\prime}(N)} c_{N, k} e^{i(k, x)}$ получаем

$$
\begin{gathered}
\nu-1-\frac{b_{\mu+\xi+1}+\cdots+b_{\nu}}{r}+(d-\nu) \frac{r+1}{r}-\frac{d}{r}-\frac{b_{\nu+1}+\cdots+b_{d}}{r}>0, \\
r(d-1)-\left(b_{\mu+\xi+1}+\cdots+b_{d}\right)-\nu>0, \\
r>\frac{\nu+b_{\mu+\xi+1}+\cdots+b_{d}}{d-1}, \\
\left\|L_{N}\right\|_{1} \gg \frac{(\log N)^{\nu-1-\frac{b_{1}+\cdots+b_{\nu}}{r}-\frac{\delta\left(\nu-d-\frac{b_{2}+\cdots+b_{\nu}}{r}\right)-\delta(\nu-1)+(d-\nu)\left(r+\frac{1}{q}\right)-d-b_{\nu+1}-\cdots-b_{d}}{r-\delta}}}{(\log N)^{\frac{\mu+\xi-1}{p^{\prime}}-\frac{b_{1}}{r}-\frac{b_{2}+\cdots+b}{\mu+\xi}}} \\
=(\log N)^{a} .
\end{gathered}
$$


Если обозначить $1-\frac{1}{p}=\delta_{1}$, то

$$
\begin{aligned}
a=\nu & -1-(\mu+\xi-1) \delta_{1}-\delta_{1} \frac{b_{2}+\cdots+b_{\mu+\xi}}{r}-\frac{b_{\mu+\xi+1}+\cdots+b_{\nu}}{r} \\
& +\frac{\delta}{r-\delta}\left(\nu-d-\frac{b_{2}+\cdots+b_{\nu}}{r}\right)-\frac{\delta(\nu-1)}{r-\delta} \\
& +(d-\nu) \frac{r+\frac{1}{q}}{r-\delta}-\frac{d}{r-\delta}-\frac{b_{\nu+1}+\cdots+b_{d}}{r-\delta} .
\end{aligned}
$$

Выбираем $p$ и $q$ так, чтобы $\delta$ и $\delta_{1}$ были достаточно малыми. Тогда с учетом условий, наложенных на $r$, получаем утверждение теоремы 3.4 .

Теоремы 3.1-3.4 показывают, что при некоторых условиях на $r$ и $b_{j}$ нельзя указать последовательность линейных ограниченных операторов, дающих приближения классов $H_{1}^{\Omega}$ в метрике $L_{1}$ и классов $H_{\infty}^{\Omega}$ в метрике $L_{\infty}$ порядка наилучшего и имеющих нормы, ограниченные в совокупности.

\section{Список литературы}

[1] В.Н. Темляков, "Приближение функций с ограниченной смешанной производной”, Тр. МИАН СССР, 178, Наука, М., 1986, 3-113; англ. пер.: V. N. Temlyakov, "Approximation of functions with a bounded mixed derivative", Proc. Steklov Inst. Math., 178 (1989), 1-121.

[2] А. С. Романюк, "Приближение классов $B_{p, \theta}^{r}$ периодических функций многих переменных линейными методами и наилучшие приближения", Матем. сб., 195:2 (2004), 91-116; англ. пер.: A. S. Romanyuk, "Approximability of the classes $B_{p, \theta}^{r}$ of periodic functions of several variables by linear methods and best approximations", Sb. Math., 195:2 (2004), 237-261.

[3] Н.Н. Пустовойтов, "Приближение многомерных функций с заданной мажорантой смешанных модулей непрерывности", Матем. заметки, 65:1 (1999), 107-117; англ. пер.: N. N. Pustovoitov, "Approximation of multidimensional functions with a given majorant of mixed moduli of continuity", Math. Notes, 65:1 (1999), 89-98.

[4] Н.Н. Пустовойтов, "Ортопоперечники классов многомерных периодических функций, мажоранта смещанных модулей непрерывности которых содержит как степенные, так и логарифмические множители", Anal. Math., 34:3 (2008), 187-224.

[5] Н. Н. Пустовойтов, "Представление и приближение периодических функций многих переменных с заданным смешанным модулем непрепывности", Anal. Math., 20:1 (1994), 35-48.

[6] К.И. Бабенко, "О приближении одного класса периодических функций многих переменных тригонометрическими многочленами", Докл. АН CCCP, 132:5 (1960), 982-985; англ. пер.: K. I. Babenko, "Approximation by trigonometric polynomials in a certain class of periodic functions of several variables", Soviet Math. Dokl., 1 (1960), 672-675.

[7] С.А. Теляковский, "Об оценках производных тригонометрических полиномов многих переменных", Сиб. матем. журнал, 4:6 (1963), 1404-1411.

[8] С.А. Теляковский, "Некоторые оценки для тригонометрических рядов с квазивыпуклыми коэффициентами", Матем. сб., 63(105):3 (1964), 426-444; англ. пер.: S. A. Telyakovskij, "Some estimates for trigonometric series with quasi-convex coefficients", Amer. Math. Soc. Transl., Ser. 2, 86 (1970), 109-131. 
[9] Б. С. Митягин, "Приближение функций в пространствах $L^{p}$ и $C$ на торе", Maтем. сб., 58(100):4 (1962), 397-414.

[10] Н. С. Никольская, "Приближение дифференцируемых функций многих переменных суммами Фурье в метрике $L_{p}$ ", Сиб. матем. журн., 15:2 (1974), 395-412; англ. пер.: N.S. Nikol'skaya, "The approximation in the $L_{p}$ metric of differentiable functions of several variables by Fourier sums", Siberian Math. J., 15:2 (1974), 282-295.

[11] Э. С. Белинский, Э. М. Галеев, “О наименьшей величине норм смешанных производных тригонометрических полиномов с заданным числом гармоник”, Beстн. Моск. ун-та. Сер. 1. Матем., мех., 1991, № 2, 3-7; англ. пер.: Eh. S. Belinskij, Eh. M. Galeev, "On the minimum of norms of mixed derivatives of trigonometric polynomials with given number of harmonics", Mosc. Univ. Math. Bull., 46:2 (1991), $4-7$.

[12] М.Б. Сихов, "Неравенства типа Бернштейна, Джексона-Никольского и оценки норм производных ядер Дирихле", Матем. заметки, 80:1 (2006), 95-104; англ. пер.: М. В. Sikhov, "Inequalities of Bernstein and Jackson-Nikol'skii type and estimates of the norms of derivatives of Dirichlet kernels", Math. Notes, 80:1 (2006), 91-100.

[13] С. М. Никольский, "Неравенства для целых функций конечной степени и их применение в теории дифференцируемых функций многих переменных", Тр. МИАН CССР, 38, Изд-во АН СССР, М., 1951, 244-278.

[14] Э. М. Галеев, "Приближение суммами Фурье классов функций с несколькими ограниченными производными”, Матем. заметки, 23:2 (1978), 197-212; англ. пер.: Е.M. Galeev, "Approximation by Fourier sums of classes of functions with several bounded derivatives", Math. Notes, 23:2 (1978), 109-117.

[15] Э. С. Белинский, "Две экстремальные задачи для тригонометрических полиномов с заданным числом гармоник", Матем. заметки, 49:1 (1991), 12-18; англ. пер.: É.S. Belinskii, "Two extremal problems for trigonometric polynomials with a prescribed number of harmonics", Math. Notes, 49:1 (1991), 10-14.

\author{
Н. Н. Пустовойтов (N. N. Pustovoitov) \\ Московский государственный технический \\ университет "МАМИ" \\ E-mail: pustovoitovnick@mail.ru
}

Поступила в редакцию 18.02.2010 и 08.06 .2011 